\title{
ESTUDO DA CARCAÇA DE MACHOS BRAFORD DESMAMADOS AOS 72 OU 210 DIAS, ABATIDOS AOS CATORZE MESES ${ }^{1}$
}

\author{
JOÃO RESTLE², FABIANO NUNES VAZ33, IVAN LUIZ BRONDANI ${ }^{4}$, \\ JORGE MACHADO GONÇALVES ${ }^{5}$ e EVELISE ANDREATTA ${ }^{6}$
}

\begin{abstract}
RESUMO - O objetivo deste trabalho foi comparar as características de carcaça de machos desmamados em duas idades, aos 72 (T72) e aos 210 (T210) dias. Utilizaram-se 36 terneiros 5/8 Hereford e $3 / 8$ Nelore, não castrados, os quais foram terminados em confinamento e abatidos aos 14 meses de idade. Não houve diferença significativa quanto ao peso de fazenda (T72=424 kg e T210=406 kg), rendimento de carcaça quente (T72 $=54,7 \%$ e T210 $=54,4 \%)$, peso de carcaça quente (T72 $=232 \mathrm{~kg}$ e $\mathrm{T} 210=221 \mathrm{~kg})$ e fria $(\mathrm{T} 72=224 \mathrm{~kg}$ e T210=214 kg) entre os dois grupos. Os animais desmamados aos 72 dias apresentaram carcaça com conformação ligeiramente superior ( 11,8 pontos vs. 11,3 pontos) e maior percentagem de costilhar em relação aos novilhos desmamados aos 210 dias, cujos cortes dianteiro e traseiro não diferiram significativamente. Não houve diferença significativa entre as variáveis comprimento de carcaça, comprimento de perna, comprimento de braço, perímetro de braço e espessura de coxão. A espessura de gordura de cobertura não diferiu significativamente entre os dois grupos de carcaça, sendo 4,6 mm e 4,2 mm, respectivamente para T72 e T210 dias. Os resultados mostraram que animais desmamados aos 72 dias de idade apresentam carcaça comparável aos desmamados com 210 dias, com peso e acabamento dentro dos limites exigidos pelos frigoríficos.
\end{abstract}

Termos para indexação: desmame precoce, gordura de cobertura, Hereford, Nelore.

\author{
CARCASS STUDY OF BRAFORD MALES \\ WEANED AT 72 OR 210 DAYS, SLAUGHTERED AT FOURTEEN MONTHS
}

\begin{abstract}
The objective of this experiment was to compare the carcass characteristics of males weaned at two ages: 72 (T72) and 210 days (T210). Thirty-six 5/8 Hereford 3/8 Nellore calves, not castrated, finished in feedlot and slaughtered at 14 months of age, were used. No significant differences were observed between treatments for final weight (T72 $=424 \mathrm{~kg}$ and T210 $=406 \mathrm{~kg}$ ), dressing percentage $(\mathrm{T} 72=54.7 \%$ and $\mathrm{T} 210=54.4 \%)$, hot carcass weight $(\mathrm{T} 72=232 \mathrm{~kg}$ and $\mathrm{T} 210=221 \mathrm{~kg})$ and cold carcass weight $(\mathrm{T} 72=224 \mathrm{~kg}$ and $\mathrm{T} 210=214 \mathrm{~kg}$ ). T72 carcasses showed better conformation (11.8 points vs 11.3 points) and higher sidecut percentage than T210, both having similar sawcut and hindquarter percentages. No significant differences were observed for carcass, leg and arm length, arm perimeter, cushion thickness and subcutaneous fat thickness (T72 $=4.6 \mathrm{~mm}$ and T210 $=4.2 \mathrm{~mm}$ ). Results demonstrate that calves weaned at 72 days of age showed similar carcasses than calves weaned at 210 days, with adequate weight and fat thickness for slaughter.
\end{abstract}

Index terms: early weaning, subcutaneus fat, Hereford, Nellore.

\footnotetext{
${ }^{1}$ Aceito para publicação em 5 de janeiro de 1999.

${ }^{2}$ Eng. Agr., Ph.D., Prof. Titular, Dep. de Zootecnia, Universidade Federal de Santa Maria (UFSM) Campus Camobi, CEP 97119-900 Santa Maria, RS. E-mail: jorestle@infoway.com.br

${ }^{3}$ Zoot., Aluno do Curso de Mestrado, UFSM.

${ }^{4}$ Zoot., M.Sc., Prof. Assistente, Dep. de Zootecnia, UFSM.

${ }^{5}$ Zoot., UFSM. Bolsista do CNPq.

${ }^{6}$ Aluna do Curso de Medicina Veterinária, UFSM. Bolsista PIBIC-CNPq.
}

\section{INTRODUÇão}

Aliado ao incremento na taxa de natalidade do rebanho, a redução da idade de abate dos machos e entoure das novilhas são metas que precisam ser atingidas para que ocorra um aumento dos índices produtivos da pecuária brasileira. Referindo-se ao confinamento para a terminação de terneiros aos quatorze meses, Restle (1997) cita que esse manejo permite que se alcance peso de carcaça e acabamen- 
to dentro dos limites exigidos pelos frigoríficos e legislações de incentivo ao abate de animais jovens (Rio Grande do Sul, 1995), como ficou demonstrado nos trabalhos de Flores (1997) e Restle \& Vaz (1997).

Atualmente nenhuma alternativa de manejo é mais eficiente para incrementar a taxa de natalidade do rebanho do que a realização do desmame dos terneiros entre 60 e 90 dias de idade, em substituição ao desmame aos sete meses, tradicionalmente realizado nas propriedades. A prática do desmame aos 60-90 dias também melhora consideravelmente o estado corporal da vaca, o que foi demonstrado no trabalho de Makarechian et al. (1988) quando constataram que a antecipação da idade ao desmame em um mês diminui a perda de peso das vacas durante o inverno de $0,480 \mathrm{~kg} /$ dia para $0,003 \mathrm{~kg} / \mathrm{dia}$.

Estudando vacas Hereford, Aberdeen Angus e Devon, Pimentel et al. (1979) verificaram taxa de repetição de cria de $64,53 \%$ e $21,46 \%$, respectivamente, em vacas desterneiradas aos 90 e 200 dias pós-parto. Desmamando terneiros aos 101 dias de idade, Moojen et al. (1994) concluíram que esse manejo aumenta a taxa de aparecimento de cio pós-parto e melhora o estado corporal da vaca no outono. Barcellos et al. (1995), desterneirando vacas aos 70 e 120 dias pós-parto, concluíram que a retirada do terneiro precocemente possibilita maior ganho de peso das mães no pós-desmama.

Segundo alguns autores, além de melhorar os índices de repetição de cria e estado corporal da vaca, o desmame aos 60-90 dias não prejudica o desenvolvimento futuro do novilho ou novilha (Grimes \& Turner, 1991; Kozloski et al., 1995). Na literatura não são encontrados estudos conduzidos sob condições brasileiras. Este trabalho teve como objetivo estudar as características de carcaça de novilhos desmamados precocemente ou não, terminados em confinamento para o abate aos 14 meses.

\section{MATERIAL E MÉTODOS}

O experimento foi conduzido no Departamento de Zootecnia da UFSM, de janeiro a dezembro de 1996, utilizando-se 36 terneiros Braford (5/8 Hereford 3/8 Nelore), não castrados, provenientes do mesmo rebanho e distribuídos ao acaso em um dos seguintes tratamentos: T72 - terneiros com idade média de 72 dias ao desmame (20 animais); T210 - terneiros com idade média de 210 dias ao desmame (16 animais).

Do nascimento aos 72 dias, os terneiros do T72 e
T210 foram mantidos nas mesmas condições; dos 72 aos 210 dias, os terneiros do T72 foram mantidos em confinamento, recebendo uma dieta contendo, na matéria seca, $50 \%$ de silagem pré-secada de Avena strigosa e 50\% de concentrado, ao passo que os do T210 permaneceram ao pé da vaca, em campo nativo melhorado. Dos 210 dias aos 14 meses de idade os animais do T72 e T210 foram confinados nas mesmas condições de manejo e alimentação, recebendo uma dieta contendo, na matéria seca, $52 \%$ de volumoso (silagem de sorgo + silagem de milho) e $48 \%$ de concentrado (à base de grão de sorgo, farelo de soja, sal e minerais)

Antes do embarque para o frigorífico, os animais foram pesados, após um jejum de sólidos de 14 horas, obtendo-se o peso de fazenda dos animais. Após o abate, foi realizada a pesagem das carcaças e da porção glandular dos testículos dos animais. Na seqüência, as carcaças foram identificadas e levadas ao resfriamento por 24 horas a $-2^{\circ} \mathrm{C}$. Decorrido esse tempo, as carcaças foram novamente pesadas, coletados os dados de comprimento de carcaça, de perna, de braço, espessura de coxão e perímetro de braço, conforme técnica descrita por Muller (1987).

As carcaças também foram avaliadas quanto à conformação, seguindo uma escala de 1 a 18 pontos (Muller, 1987), e medida a espessura de gordura subcutânea sobre a 12 a costela, com ajuste do valor quando não era representativa da real cobertura de gordura da carcaça. Também foi medida a percentagem de corte dianteiro, traseiro (ou corte serrote) e costilhar

O delineamento experimental foi o inteiramente casualizado, com número desigual de repetições por tratamento, sendo os resultados submetidos à análise de variância, pelo método dos quadrados mínimos, utilizando o seguinte modelo matemático:

$Y_{i j}=\mu+T_{i}+e_{i j}$, em que

$Y_{i j}=$ observação $\mathrm{j}$ do animal que recebeu tratamento i;

$\mu=$ média geral;

$T_{i}=$ efeito do tratamento $\mathrm{i}$;

$e_{i j}=$ erro aleatório associado a cada observação

Também foi calculado o coeficiente de correlação entre cada variável estudada, considerando ou não os tratamentos, e verificada a probabilidade da correlação ser significativa a $5 \%$ pelo teste $t$.

\section{RESULTADOS E DISCUSSÃO}

$\mathrm{Na}$ Tabela 1 são apresentados os resultados referentes a peso de fazenda, de carcaça, rendimento, quebra ao resfriamento e peso da porção glandular dos testículos. Observa-se que não houve diferença $(\mathrm{P}>0,05)$ entre os tratamentos em relação a tais características. O peso de fazenda similar entre os dois tratamentos, revela que o desenvolvimento dos animais não foi afetado pela idade ao des- 
mame, e que não houve reflexos, também, nas características de peso de carcaça quente $(\mathrm{T} 72=232 \mathrm{~kg}$ e $\mathrm{T} 210=221 \mathrm{~kg})$ e fria $(\mathrm{T} 72=224 \mathrm{~kg}$ e T2 $10=214 \mathrm{~kg})$, e rendimento de carcaça quente $(\mathrm{T} 72=54,7 \%$ e $\mathrm{T} 210=54,4 \%)$ e fria $(\mathrm{T} 72=52,8 \%$ e T $210=52,7 \%)$

Kubisch \& Makarechian (1987), utilizando 367 terneiros inteiros, desmamados aos 157 e 189 dias de idade, verificaram que aqueles desmamados mais precocemente foram $14,3 \mathrm{~kg}$ mais leves ao abate que os desmamados com mais idade. Entretanto, Grimes \& Turner (1991) verificaram, em cinco anos de estudo, que terneiros desmamados aos 110 dias foram $14,1 \mathrm{~kg}$ mais pesados que os terneiros desmamados aos 220 dias de idade. Esses resultados, somados aos do presente estudo, demonstram que pode-se obter desenvolvimento adequado, e conseqüentemente peso de abate e de carcaça em animais desmamados precocemente, desde que não sofram restrições de meio ambiente.

Trabalhando com animais desmamados aos 120, 150,180 e 210 dias de idade, abatidos aos 18 meses, Proskuryakov et al. (1982) também não verificaram efeito da idade ao desmame sobre o rendimento de carcaça, que foi de 54,2, 54,0, 53,6 e $53,9 \%$, respectivamente. Esses dados demonstram que a teoria empírica de que animais desmamados precocemente, devido ao desenvolvimento precoce do trato digestivo, teriam pior rendimento de carcaça que animais desmamados com mais idade não se confirmou nas condições em que se conduziu o presente experimento.

$\mathrm{Na}$ Tabela 2 são apresentados os resultados referentes a comprimento de carcaça, de perna, de braço, espessura de coxão, perímetro de braço, conformação e espessura de gordura subcutânea. Observa-se que nas características de comprimento de carcaça, de braço e de perna, que refletem o desenvolvimento ósseo da carcaça, não houve diferença $(\mathrm{P}>0,05)$ entre os dois tratamentos, o mesmo verificando-se com as características de musculosidade dos membros (espessura de coxão e perímetro de braço). A conformação de carcaça foi classificada como "boa mais" (11,8 pontos) nos animais do T72, sendo ligeiramente superior à dos animais do T210, que tiveram conformação de carcaça classificada como "boa típica" (11,3 pontos), entretanto não houve diferença estatística entre tratamentos $(\mathrm{P}>0,05)$. Também não foi verificada diferença $(\mathrm{P}>0,05)$ na espessura de gordura de cobertura. Grimes \& Turner (1991), confinando animais até que atingissem $0,89 \mathrm{~cm}$ sobre a 12 a costela, verificaram que os terneiros desmamados aos 110 dias foram 11 dias mais jovens ao abate que terneiros que permaneceram ao pé da vaca até os 220 dias.

No presente trabalho, a espessura de gordura média dos dois tratamentos, sobre a $12^{a}$ costela, foi de 4,4 mm, um acabamento considerado adequado pelas legislações de incentivo ao abate de animais jovens (Rio Grande do Sul, 1995), principalmente por tratar-se de animais inteiros. Esse bom acabamento das carcaças de machos inteiros, confinados após o desmame e abatidos aos 14 meses de idade, também é comentado nos trabalhos de Flores (1997), com Hereford com diferentes graus de sangue Nelore (4,1 a 5,0 mm), e de Restle \& Vaz (1997), com Hereford (4,64 mm).

$\mathrm{Na}$ Tabela 3 são apresentados os resultados referentes à percentagem e peso dos três cortes comerciais da carcaça. Verifica-se que os animais desmamados aos 72 dias apresentaram percentagem $(\mathrm{P}<0,0470)$ e peso de costilhar $(\mathrm{P}<0,0357)$ maior que os desmamados aos 210 dias de idade. Isso pode ter

TABELA 1. Média e desvio-padrão (DP) de peso de fazenda, peso da porção glandular dos testículos, peso de carcaça quente e de carcaça fria, rendimento e quebra no resfriamento da carcaça de animais desmamados aos 72 ou 210 dias de idade.

\begin{tabular}{|c|c|c|c|c|c|c|}
\hline \multirow[t]{2}{*}{ Parâmetro } & \multicolumn{2}{|c|}{$\mathrm{T} 72$} & \multicolumn{2}{|c|}{$\mathrm{T} 210$} & \multirow[t]{2}{*}{$\mathrm{CV}^{1}(\%)$} & \multirow[t]{2}{*}{$\mathrm{P}<\mathrm{F}$} \\
\hline & Média & DP & Média & DP & & \\
\hline Peso de fazenda $(\mathrm{kg})$ & 424 & 10,42 & 406 & 9,32 & 10,07 & 0,1875 \\
\hline Peso de carcaça quente $(\mathrm{kg})$ & 232 & 5,92 & 221 & 5,29 & 10,49 & 0,1558 \\
\hline Peso de carcaça fria $(\mathrm{kg})$ & 224 & 5,96 & 214 & 5,33 & 10,92 & 0,1830 \\
\hline Rendimento carcaça quente $(\%)$ & 54,7 & 0,48 & 54,4 & 0,43 & 3,51 & 0,6903 \\
\hline Rendimento carcaça fria $(\%)$ & 52,8 & 0,46 & 52,7 & 0,41 & 3,50 & 0,7953 \\
\hline Quebra ao resfriamento $(\%)$ & 3,37 & 0,49 & 3,24 & 0,45 & 60,47 & 0,8501 \\
\hline Peso de testículo $(\mathrm{kg})$ & 0,40 & 0,04 & 0,37 & 0,03 & 32,80 & 0,6892 \\
\hline
\end{tabular}


sido resultado da diferença alimentar no período dos 72 aos 210 dias de idade, quando havia animais sendo amamentados (T210) e animais recebendo alimentação em confinamento (T72), o que pode ter levado ao acúmulo de gordura na região do costilhar. $\mathrm{O}$ coeficiente de correlação entre percentagem de costilhar e espessura de gordura foi de $0,55(\mathrm{P}<0,0260)$ no T72 (Tabela 4).

Na Tabela 5, observa-se que independentemente do tratamento, o peso de testículo teve correlação alta com o peso de fazenda $(\mathrm{r}=0,67 ; \mathrm{P}<0,0021)$, mas o coeficiente de correlação entre essas duas variáveis foi maior ainda quando estudaram-se os animais do T72 isoladamente $(\mathrm{r}=0,83 ; \mathrm{P}<0,0115$; Tabela 4). Esses resultados mostram que a produção de hormônios androgênicos tem efeito positivo no desenvolvimento do animal até os 14 meses, como ficou demonstrado por Restle \& Vaz (1997) quando estudaram animais inteiros e castrados abatidos também aos 14 meses de idade.
O peso de fazenda também apresentou coeficiente de correlação alto e significativo com as medidas de comprimento de carcaça no T72 (Tabela 4) e comprimento de braço, de perna, perímetro de braço e espessura de coxão independentemente do tratamento (Tabela 5). A mesma correlação foi observada entre cada uma das cinco medidas de desenvolvimento estrutural da carcaça e peso de carcaça fria. $\mathrm{O}$ peso de carcaça fria também apresentou coeficiente de correlação significativo com a conformação de carcaça $(\mathrm{r}=0,45 ; \mathrm{P}<0,0060)$ e espessura de gordura $(\mathrm{r}=0,40 ; \mathrm{P}<0,0151)$, o que também é citado por Müller (1987), que comenta que em carcaças de mesmo comprimento e espessura de gordura, as carcaças de melhor conformação apresentam maior peso.

$\mathrm{Na}$ análise do rendimento de carcaça, os coeficientes de correlação foram altos e significativos entre essa característica e a conformação e espessura de gordura, no T72, mas foram baixos e não significa-

TABELA 2. Média e desvio-padrão (DP) de medidas de carcaça, conformação e espessura de gordura de cobertura na carcaça de animais desmamados aos 72 ou 210 dias de idade.

\begin{tabular}{|c|c|c|c|c|c|c|}
\hline \multirow[t]{2}{*}{ Parâmetro } & \multicolumn{2}{|c|}{$\mathrm{T} 72$} & \multicolumn{2}{|c|}{$\mathrm{T} 210$} & \multirow[t]{2}{*}{$\mathrm{CV}^{1}(\%)$} & \multirow[t]{2}{*}{$\mathrm{P}<\mathrm{F}$} \\
\hline & Média & $\mathrm{DP}$ & Média & DP & & \\
\hline Comprimento carcaça $(\mathrm{cm})$ & 118,8 & 1,45 & 117,7 & 1,30 & 4,91 & 0,5717 \\
\hline Comprimento perna $(\mathrm{cm})$ & 69,0 & 6,92 & 69,6 & 6,19 & 3,99 & 0,5227 \\
\hline Espessura coxão $(\mathrm{cm})$ & 23,8 & 5,31 & 23,8 & 4,75 & 8,92 & 0,9861 \\
\hline Comprimento braço $(\mathrm{cm})$ & 37,6 & 4,49 & 37,2 & 4,02 & 4,80 & 0,6073 \\
\hline Perímetro braço $(\mathrm{cm})$ & 36,3 & 4,50 & 36,1 & 4,03 & 4,98 & 0,6668 \\
\hline Conformação ${ }^{2}$ & 11,8 & 0,35 & 11,3 & 0,31 & 12,03 & 0,2876 \\
\hline Espessura gordura (mm) & 4,6 & 4,54 & 4,2 & 4,06 & 41,54 & 0,5227 \\
\hline
\end{tabular}

${ }^{1}$ Coeficiente de variação.

${ }^{2}$ Valor $10=$ boa menos; $11=$ boa típica; $12=$ boa mais

TABELA 3. Média e desvio-padrão (DP) de peso e percentagem dos três cortes comerciais da carcaça de animais desmamados aos 72 ou 210 dias de idade.

\begin{tabular}{|c|c|c|c|c|c|c|}
\hline \multirow[t]{2}{*}{ Parâmetro } & \multicolumn{2}{|c|}{$\mathrm{T} 72$} & \multicolumn{2}{|c|}{$\mathrm{T} 210$} & \multirow[t]{2}{*}{$\mathrm{CV}^{1}(\%)$} & \multirow[t]{2}{*}{$\mathrm{P}<\mathrm{F}$} \\
\hline & Média & DP & Média & DP & & \\
\hline Peso de dianteiro $(\mathrm{kg})$ & 43,8 & 1,17 & 42,2 & 1,05 & 10,92 & 0,3152 \\
\hline Percentagem de dianteiro & 37,0 & 0,33 & 37,8 & 0,29 & 3,55 & 0,0907 \\
\hline Peso de costilhar (kg) & 15,3 & 0,62 & 13,5 & 0,56 & 17,46 & 0,0357 \\
\hline Percentagem de costilhar & 12,8 & 0,29 & 12,0 & 0,26 & 9,34 & 0,0470 \\
\hline Peso de traseiro $(\mathrm{kg})$ & 59,5 & 1,45 & 56,0 & 1,29 & 10,08 & 0,0869 \\
\hline Percentagem de traseiro & 50,2 & 0,34 & 50,2 & 0,30 & 2,68 & 0,9591 \\
\hline
\end{tabular}


TABELA 4. Coeficiente de correlação entre as variáveis estudadas da carcaça de animais desmamados aos 72 dias de idade.

\begin{tabular}{|c|c|c|c|c|c|c|c|c|c|c|c|c|c|c|c|c|}
\hline Variável & & PCF & RCQ & RCF & $\mathrm{QR}$ & PTest & $\mathrm{CC}$ & $\mathrm{CP}$ & EC & $\mathrm{CB}$ & $\mathrm{PB}$ & Co & EG & $\mathrm{PD}$ & $\mathrm{PC}$ & $\mathrm{PTr}^{1}$ \\
\hline \multirow[t]{2}{*}{ Peso de fazenda } & $\mathrm{r}$ & 0,98 & 0,06 & 0,32 & $-0,31$ & 0,83 & 0,84 & 0,64 & 0,61 & 0,65 & 0,73 & 0,34 & 0,30 & $-0,31$ & 0,54 & $-0,30$ \\
\hline & Prob. & 0,0001 & 0,8169 & 0,2279 & 0,2398 & 0,0115 & 0,0001 & 0,0078 & 0,0117 & 0,0066 & 0,0014 & 0,1987 & 0,2493 & 0,2374 & 0,0295 & 0,2545 \\
\hline \multirow{2}{*}{$\begin{array}{l}\text { Peso carcaça fria } \\
\text { (PCF) }\end{array}$} & $\mathrm{r}$ & & 0,22 & 0,50 & $-0,27$ & 0,84 & 0,82 & 0,58 & 0,68 & 0,63 & 0,77 & 0,41 & 0,36 & $-0,36$ & 0,56 & $-0,28$ \\
\hline & Prob. & & 0,4037 & 0,0488 & 0,3033 & 0,0094 & 0,0001 & 0,0177 & 0,0039 & 0,0092 & 0,0004 & 0,1135 & 0,1636 & 0,1710 & 0,0253 & 0,3009 \\
\hline \multirow{2}{*}{$\begin{array}{l}\text { Rend. carc. quente } \\
\text { (RCQ) }\end{array}$} & $\mathrm{r}$ & & & 0,80 & 0,6342 & 0,25 & 0,01 & $-0,42$ & 0,23 & 0,04 & 0,44 & 0,63 & 0,59 & $-0,30$ & 0,18 & 0,06 \\
\hline & Prob. & & & 0,0002 & 0,0083 & 0,5457 & 0,9807 & 0,1023 & 0,3962 & 0,8871 & 0,0886 & 0,0090 & 0,0155 & 0,2608 & 0,4949 & 0,8205 \\
\hline \multirow{2}{*}{$\begin{array}{l}\text { Rend. carc. fria } \\
\text { (RCF) }\end{array}$} & $\mathrm{r}$ & & & & 0,05 & 0,57 & 0,24 & 0,01 & 0,59 & 0,16 & 0,54 & 0,47 & 0,41 & $-0,37$ & 0,30 & 0,00 \\
\hline & Prob. & & & & 0,8532 & 0,1395 & 0,3751 & 0,9785 & 0,0159 & 0,5632 & 0,0297 & 0,0645 & 0,1182 & 0,1621 & 0,2661 & 0,9920 \\
\hline \multirow{2}{*}{$\begin{array}{l}\text { Quebra resfriam. } \\
(\mathrm{QR})\end{array}$} & $\mathrm{R}$ & & & & & $-0,91$ & $-0,30$ & $-0,72$ & $-0,39$ & $-0,14$ & 0,03 & 0,45 & 0,45 & $-0,03$ & $-0,08$ & 0,11 \\
\hline & Prob. & & & & & 0,0019 & 0,2545 & 0,0015 & 0,1373 & 0,6073 & 0,9092 & 0,0831 & 0,0715 & 0,9201 & 0,7652 & 0,6906 \\
\hline \multirow{4}{*}{$\begin{array}{l}\text { Peso testículo } \\
\text { (PTest) } \\
\text { Compr. carcaça } \\
\text { (CC) }\end{array}$} & $\mathrm{r}$ & & & & & & 0,86 & 0,57 & 0,60 & 0,68 & 0,90 & 0,54 & 0,45 & $-0,30$ & 0,67 & $-0,36$ \\
\hline & Prob. & & & & & & 0,0054 & 0,1351 & 0,1134 & 0,0624 & 0,0020 & 0,1684 & 0,2629 & 0,4708 & 0,0669 & 0,3760 \\
\hline & $\mathrm{r}$ & & & & & & & 0,62 & 0,34 & 0,78 & 0,46 & 0,12 & 0,14 & $-0,01$ & 0,48 & $-0,49$ \\
\hline & Prob. & & & & & & & 0,0103 & 0,2012 & 0,0004 & 0,0703 & 0,6442 & 0,6072 & 0,9812 & 0,0613 & 0,0513 \\
\hline Compr. perna & $\mathrm{r}$ & & & & & & & & 0,54 & 0,61 & 0,22 & $-0,32$ & $-0,30$ & 0,11 & 0,10 & $-0,19$ \\
\hline (CP) & Prob. & & & & & & & & 0,0293 & 0,0111 & 0,4068 & 0,2230 & 0,2592 & 0,6959 & 0,7146 & 0,4710 \\
\hline Espess. coxão & $\mathrm{r}$ & & & & & & & & & 0,26 & 0,48 & 0,17 & 0,21 & 0,40 & 0,45 & $-0,13$ \\
\hline (EC) & Prob. & & & & & & & & & 0,3317 & 0,0577 & 0,5163 & 0,4393 & 0,1252 & 0,0783 & 0,6210 \\
\hline Compr. braço & $\mathrm{r}$ & & & & & & & & & & 0,38 & 0,05 & $-0,04$ & 0,20 & 0,08 & $-0,25$ \\
\hline (CB) & Prob. & & & & & & & & & & 0,1458 & 0,8569 & 0,8844 & 0,4600 & 0,7813 & 0,3535 \\
\hline Perím. braço & $\mathrm{r}$ & & & & & & & & & & & 0,54 & 0,36 & $-0,27$ & 0,27 & $-0,05$ \\
\hline$(\mathrm{PB})$ & Prob. & & & & & & & & & & & 0,0302 & 0,1648 & 0,3139 & 0,3165 & 0,8507 \\
\hline Conformação & $\mathrm{r}$ & & & & & & & & & & & & 0,78 & $-0,55$ & 0,30 & 0,15 \\
\hline (Co) & Prob. & & & & & & & & & & & & 0,0004 & 0,0287 & 0,2513 & 0,5901 \\
\hline Espessura gordura & $\mathrm{r}$ & & & & & & & & & & & & & $-0,56$ & 0,55 & $-0,10$ \\
\hline (EG) & Prob. & & & & & & & & & & & & & 0,0243 & 0,0260 & 0,7017 \\
\hline Perc. dianteiro & $\mathrm{r}$ & & & & & & & & & & & & & & $-0,46$ & $-0,37$ \\
\hline (PD) & Prob. & & & & & & & & & & & & & & 0,0724 & 0,1597 \\
\hline Perc. costilhar & $\mathrm{r}$ & & & & & & & & & & & & & & & $-0,65$ \\
\hline (PC) & Prob. & & & & & & & & & & & & & & & 0,0059 \\
\hline
\end{tabular}




\begin{tabular}{|c|c|c|c|c|c|c|c|c|c|c|c|c|c|c|c|c|}
\hline Variável & & PCF & $\mathrm{RCQ}$ & $\mathrm{RCF}$ & $\mathrm{QR}$ & PTest & $\mathrm{CC}$ & $\mathrm{CP}$ & EC & $\mathrm{CB}$ & $\mathrm{PB}$ & $\mathrm{Co}$ & EG & $\mathrm{PD}$ & $\mathrm{PC}$ & $\mathrm{PTr}^{1}$ \\
\hline \multirow[t]{2}{*}{ Peso de fazenda } & $\mathrm{r}$ & 0,95 & $-0,05$ & 0,08 & $-0,23$ & 0,67 & 0,69 & 0,60 & 0,52 & 0,56 & 0,66 & 0,29 & 0,28 & $-0,15$ & 0,15 & 0,02 \\
\hline & Prob. & 0,0001 & 0,7782 & 0,6234 & 0.1768 & 0,0021 & 0.0001 & 0.0001 & 0.0012 & 0.0003 & 0,0001 & 0.0867 & 0.0951 & 0,3694 & 0.3732 & 0,9102 \\
\hline Peso carcaça fria & $\mathrm{r}$ & & 0,27 & 0,34 & $-0,12$ & 0,61 & 0,63 & 0,48 & 0,57 & 0,49 & 0,69 & 0,45 & 0,40 & $-0,17$ & 0,50 & $-0,28$ \\
\hline (PCF) & Prob. & & 0,1052 & 0,0397 & 0,4810 & 0,0074 & 0,0001 & 0,0031 & 0,0003 & 0,0025 & 0,0001 & 0,0060 & 0,0151 & 0,3189 & 0,0019 & 0,0981 \\
\hline Rend. carc. Ouente & $\mathrm{r}$ & & & 0,38 & $-0,30$ & 0,62 & 0,65 & 0,56 & 0,64 & 0,48 & 0,64 & 0,36 & 0,31 & $-0,16$ & 0,51 & $-0,30$ \\
\hline (RCQ) & Prob. & & & 0,0221 & 0,0792 & 0,0065 & 0,0001 & 0,0003 & 0,0001 & 0,0026 & 0,0001 & 0,0312 & 0,0643 & 0,3610 & 0,0015 & 0,0719 \\
\hline Rend. carc. Fria & $\mathrm{r}$ & & & & 0,29 & $-0,01$ & $-0,08$ & $-0,28$ & 0,22 & $-0,16$ & 0,17 & 0,50 & 0,37 & $-0,02$ & 0,03 & $-0,01$ \\
\hline (RCF) & Prob. & & & & 0,0816 & 0,9762 & 0,6232 & 0,1036 & 0,2031 & 0,3586 & 0,3331 & 0,0021 & 0,0260 & 0,9254 & 0,8761 & 0,9636 \\
\hline Quebra resfriam. & $\mathrm{R}$ & & & & & 0,11 & 0,04 & 0,04 & 0,53 & $-0,11$ & 0,09 & 0,27 & 0,14 & 0,01 & 0,12 & $-0,12$ \\
\hline (QR) & Prob. & & & & & 0,6571 & 0.7960 & 0.7990 & 0.0010 & 0.5065 & 0,5819 & 0.1133 & 0.4130 & 0,9448 & 0.4839 & 0,4782 \\
\hline Peso testículo & $\mathrm{r}$ & & & & & & $-0,22$ & $-0,54$ & $-0,53$ & $-0,07$ & 0,12 & 0,38 & 0,39 & $-0,06$ & $-0,16$ & 0,21 \\
\hline (PTest) & Prob. & & & & & & 0.1960 & 0.0006 & 0.0010 & 0.6960 & 0,4697 & 0.0206 & 0.0198 & 0,7471 & 0.3448 & 0,2303 \\
\hline Compr. Carcaça & $\mathrm{r}$ & & & & & & & 0,39 & 0,37 & 0,33 & 0,74 & 0,02 & 0,47 & $-0,29$ & 0,63 & $-0,21$ \\
\hline (CC) & Prob. & & & & & & & 0.1067 & 0.1263 & 0.1840 & 0,0004 & 0.9241 & 0.0475 & 0,2436 & 0.0052 & 0,3964 \\
\hline Compr. Perna & $\mathrm{r}$ & & & & & & & & 0,22 & 0,58 & 0,35 & $-0,03$ & 0,21 & $-0,12$ & 0,43 & $-0,27$ \\
\hline$(\mathrm{CP})$ & Prob. & & & & & & & & 0,1937 & 0,0002 & 0,0358 & 0,8466 & 0,2117 & 0,4990 & 0,0097 & 0,1148 \\
\hline Espess. Coxão & $\mathrm{r}$ & & & & & & & & & 0,55 & 0,25 & $-0,26$ & $-0,19$ & $-0,00$ & 0,22 & $-0,20$ \\
\hline (EC) & Prob. & & & & & & & & & 0,0006 & 0,1492 & 0,1246 & 0,2513 & 0,9935 & 0,2050 & 0,2529 \\
\hline Compr. Braço & $\mathrm{r}$ & & & & & & & & & & 0,24 & 0,08 & $-0,01$ & $-0,11$ & 0,27 & $-0,13$ \\
\hline (CB) & Prob. & & & & & & & & & & 0,1603 & 0,6344 & 0,9432 & 0,5141 & 0,1059 & 0,4366 \\
\hline Perím. Braço & $\mathrm{r}$ & & & & & & & & & & & $-0,12$ & 0,00 & $-0,01$ & 0,18 & $-0,15$ \\
\hline (PB) & Prob. & & & & & & & & & & & 0.4737 & 0.9897 & ,09371 & 0.2881 & 0,3771 \\
\hline Conformação & $\mathrm{r}$ & & & & & & & & & & & & 0,34 & $-0,01$ & 0,18 & $-0,15$ \\
\hline (Co) & Prob. & & & & & & & & & & & & 0.0421 & 0,9371 & 0.2881 & 0,3771 \\
\hline Espessura gordura & $\mathrm{r}$ & & & & & & & & & & & & & $-0,07$ & 0,08 & 0,00 \\
\hline (EG) & Prob. & & & & & & & & & & & & & 0,9769 & 0,6508 & 0,9864 \\
\hline Perc. Dianteiro & $\mathrm{r}$ & & & & & & & & & & & & & & 0,44 & $-0,06$ \\
\hline (PD) & Prob. & & & & & & & & & & & & & & 0,0074 & 0,7453 \\
\hline Perc. Costilhar & $\mathrm{r}$ & & & & & & & & & & & & & & & $-0,60$ \\
\hline (PC) & Prob. & & & & & & & & & & & & & & & 0,0001 \\
\hline
\end{tabular}


TABELA 6. Coeficiente de correlação entre as variáveis estudadas da carcaça de animais desmamados aos 210 dias de idade.

\begin{tabular}{|c|c|c|c|c|c|c|c|c|c|c|c|c|c|c|c|c|}
\hline Variável & & PCF & RCQ & $\mathrm{RCF}$ & QR & PTest & $\mathrm{CC}$ & $\mathrm{CP}$ & EC & $\mathrm{CB}$ & PB & Co & EG & $\mathrm{PD}$ & PC & $\mathrm{PTr}^{1}$ \\
\hline Peso de fazenda & $\mathrm{r}$ & 0,89 & $-0,22$ & 0,10 & $-0,18$ & 0,40 & 0,33 & 0,66 & 0,42 & 0,40 & 0,57 & 0,16 & 0,18 & 0,02 & 0,39 & $-0,32$ \\
\hline & Prob. & 0,0001 & 0,3491 & 0,6620 & 0,4356 & 0,2543 & 0,1585 & 0,0014 & 0,0622 & 0,0768 & 0,0088 & 0,4940 & 0,4384 & 0,9169 & 0,0888 & 0,1751 \\
\hline Peso carcaça fria & $\mathrm{r}$ & & 0,18 & 0,36 & $-0,38$ & 0,20 & 0,24 & 0,66 & 0,64 & 0,21 & 0,45 & 0,24 & 0,15 & 0,10 & 0,37 & $-0,38$ \\
\hline (PCF) & Prob. & & 0,4365 & 0,1208 & 0,0971 & 0,5849 & 0,3019 & 0,0016 & 0,0025 & 0,3611 & 0,0479 & 0,3083 & 0,5204 & 0,6712 & 0,1087 & 0,0979 \\
\hline Rend. carc. quente & $\mathrm{r}$ & & & 0,86 & 0,06 & $-0,21$ & $-0,24$ & $-0,12$ & 0,21 & $-0,38$ & $-0,08$ & 0,39 & 0,13 & 0,15 & $-0,14$ & $-0,05$ \\
\hline$(\mathrm{RCQ})$ & Prob. & & & 0,0001 & 0,7843 & 0,5654 & 0,3083 & 0,6005 & 0,3690 & 0,0948 & 0,7425 & 0,0879 & 0,5758 & 0,5351 & 0,5431 & 0,8416 \\
\hline Rend. carc. fria & $\mathrm{r}$ & & & & $-0,46$ & $-0,16$ & $-0,13$ & 0,09 & 0,53 & $-0,33$ & $-0,17$ & 0,16 & $-0,06$ & 0,16 & 0,01 & $-0,17$ \\
\hline (RCF) & Prob. & & & & 0,0421 & 0,6531 & 0,5737 & 0,7139 & 0,0168 & 0,1501 & 0,4583 & 0,4846 & 0,8141 & 0,5120 & 0,9551 & 0,4640 \\
\hline Quebra resfriam. & $\mathrm{r}$ & & & & & $-0,27$ & $-0,15$ & $-0,38$ & $-0,66$ & $-0,00$ & 0,21 & 0,34 & 0,33 & $-0,06$ & $-0,27$ & 0,26 \\
\hline (QR) & Prob. & & & & & 0,4352 & 0,5231 & 0,1017 & 0,0017 & 0,9921 & 0,3846 & 0,1435 & 0,1572 & 0,8031 & 0,2469 & 0,2610 \\
\hline Peso testículo & $\mathrm{r}$ & & & & & & 0,64 & 0,39 & 0,05 & 0,11 & 0,51 & $-0,43$ & 0,49 & $-0,29$ & 0,64 & $-0,09$ \\
\hline (PTest) & Prob. & & & & & & 0,0474 & 0,2593 & 0,8965 & 0,7649 & 0,1252 & 0,2196 & 0,1460 & 0,4238 & 0,0483 & 0,8025 \\
\hline Compr. carcaça & $\mathrm{r}$ & & & & & & & 0,41 & 0,03 & 0,15 & 0,15 & $-0,34$ & 0,36 & $-0,20$ & 0,35 & $-0,05$ \\
\hline (CC) & Prob. & & & & & & & 0,0750 & 0,8810 & 05366 & 0,5126 & 0,1404 & 0,1133 & 0,3902 & 0,1301 & 0,8470 \\
\hline Compr. perna & $\mathrm{r}$ & & & & & & & & 0,21 & 0,47 & 0,30 & $-0,16$ & 0,03 & $-0,15$ & 0,50 & $-0,22$ \\
\hline$(\mathrm{CP})$ & Prob. & & & & & & & & 0,3664 & 0,0357 & 0,1914 & 0,5073 & 0,9099 & 0,5376 & 0,0243 & 0,3560 \\
\hline Espess. coxão & $\mathrm{r}$ & & & & & & & & & $-0,11$ & $-0,04$ & $-0,01$ & $-0,35$ & 0,05 & 0,11 & $-0,14$ \\
\hline (EC) & Prob. & & & & & & & & & 0,6476 & 0,8743 & 0,9663 & 0,1305 & 0,8330 & 0,6310 & 0,5625 \\
\hline Compr. braço & $\mathrm{r}$ & & & & & & & & & & 0,49 & $-0,38$ & 0,05 & $-0,13$ & 0,28 & $-0,07$ \\
\hline$(\mathrm{CB})$ & Prob. & & & & & & & & & & 0,0271 & 0,0988 & 0,8295 & 0,5979 & 0,2357 & 0,7564 \\
\hline Perím. braço & $\mathrm{r}$ & & & & & & & & & & & $-0,08$ & 0,30 & 0,18 & 0,07 & $-0,24$ \\
\hline$(\mathrm{PB})$ & Prob. & & & & & & & & & & & 0,7426 & 0,2018 & 0,4572 & 0,7777 & 0,3181 \\
\hline Conformação & $\mathrm{r}$ & & & & & & & & & & & & 0,27 & 0,27 & $-0,26$ & $-0,09$ \\
\hline (Co) & Prob. & & & & & & & & & & & & 0,2464 & 0,2449 & 0,2666 & 0,6965 \\
\hline Espessura gordura & $\mathrm{r}$ & & & & & & & & & & & & & $-0,17$ & 0,26 & $-0,01$ \\
\hline (EG) & Prob. & & & & & & & & & & & & & 0,4654 & 0,2663 & 0,9613 \\
\hline Perc. dianteiro & $\mathrm{r}$ & & & & & & & & & & & & & & $-0,42$ & $-0,74$ \\
\hline (PD) & Prob. & & & & & & & & & & & & & & 0,0654 & 0,0002 \\
\hline Perc. costilhar & $\mathrm{r}$ & & & & & & & & & & & & & & & $-0,30$ \\
\hline (PC) & Prob. & & & & & & & & & & & & & & & 0,1982 \\
\hline
\end{tabular}


tivos no T210. O coeficiente de correlação foi de 0,53 entre rendimento de carcaça fria e espessura de coxão $(\mathrm{P}<0,0168)$ no T210 (Tabela 6), que assim como a conformação é uma medida de musculosidade da carcaça. $\mathrm{O}$ rendimento de carcaça quente foi significativamente correlacionado com a conformação $(r=0,63$; $\mathrm{P}<0,0090)$ e espessura de gordura $(\mathrm{r}=0,59 ; \mathrm{P}<0,0155)$ no T72, mas não apresentou correlação significativa quando foram estudados apenas os animais do T210.

\section{CONCLUSÕES}

1. O desmame de terneiros aos 72 dias de idade e a manutenção destes sob bons níveis nutricionais, quando comparado ao desmame aos 210 dias não afeta as características de carcaça aos 14 meses de idade, exceto peso e percentagem de costilhar, que é maior nos terneiros desmamados com 72 dias.

2. O peso da porção glandular dos testículos correlaciona-se positivamente com as características peso de fazenda e de desenvolvimento da carcaça.

3. A terminação em confinamento, independentemente da época de desmame, permite que se atinja, aos 14 meses de idade, peso de carcaça e acabamento dentro dos padrões exigidos pelos frigoríficos do Rio Grande do Sul, mesmo em animais inteiros.

\section{REFERÊNCIAS}

BARCELLOS, J.O.J.; LOBATO, J.F.P.; SILVA, M.D Desempenho ponderal de vacas e terneiros de corte submetidos ao desmame aos 70 ou 120 dias de idade. In: REUNIÃO ANUAL DA SOCIEDADE BRASILEIRA DE ZOOTECNIA, 32., 1995 , Brasília. Anais. Brasília: SBZ, 1995. p.426-427.

FLORES, J.L.C. Desempenho em confinamento de terneiros inteiros de diferentes grupos genéticos na fase do desmame ao abate aos quatorze meses. Santa Maria: UFSM, 1997. 136p. Dissertação de Mestrado

GRIMES. J.F.; TURNER, T.B. Early weaning of fall-born beef calves. 2. Postweaning performance of early and normal weaned calves. Journal of Production Agriculture, Columbus, v.4, n.4, p.468-471, Apr. 1991.

KOZLOSKI, G.V.; SANCHEZ, L.M.B.; BOETCHER, A. Alimentação de terneiros com dietas ricas em grãos e suplementados com uréia ou farinha de carne.
I. Ganho de peso e consumo de alimento, In: REUNIÃO ANUAL DA SOCIEDADE BRASILEIRA DE ZOOTECNIA, 32., 1995, Brasília. Anais. Brasília: SBZ, 1995. p.201-202.

KUBISCH, H.M.; MAKARECHIAN, M. Effects of date of weaning on postweaning performance of bull calves of three breed groups. Canadian Journal of Animal Science, Lacombe, v.67, n.4, p.941-949, Apr. 1987

MAKARECHIAN, M.; KUBISCH, H.M.; PRICE, M.A. Effects of date of weaning on subsequent performance of beef cows and their female calves. Canadian Journal of Animal Science, Lacombe, v.68, n.4, p.1035-1040, Apr. 1988.

MOOJEN, J.G.; RESTLE, J.; MOOJEN, E.L. Efeito da época da desmama e da pastagem no desempenho de vacas e terneiros de corte. 1. Desempenho das vacas. Ciência Rural, Santa Maria, v.24, n.2, p.393-397, abr./jun. 1994.

MULLER, L. Normas para avaliação de carcaças e concurso de carcaças de novilhos. 2.ed. Santa Maria: UFSM Imprensa Universitária, 1987. 31p.

PIMENTEL, C.A.; DESCHAMPS, J.C.; OLIVEIRA, J.A.F.; CARDELINO, R.; PIMENTEL, M.A. Effects of early weaning on reproductive efficiency in beef cows. Theriogenology, v.11, n.6, p.421-427, June 1979.

PROSKURYAKOV, A.; IBRAGIMOV, U.; KHARITONOVA, S. Weaning age of young beef cattle during intensive rearing. Molochnoe i Myasnoe Skotovodstvo, Moscou, n.9, p.29-30, Sept. 1982

RESTLE, J. Confinamento de terneiros. In: RESTLE, J.; BRONDANI, I.L.; PASCOAL, L.L.; LUPATINI, G.C.; VAZ, F.N. (Eds.). Técnicas avançadas na recria e engorda de bovinos de corte. Santa Maria: UFSM, 1997. p.51-67.

RESTLE, J.; VAZ, F.N. Aspectos quantitativos da carcaça de machos Hereford, inteiros ou castrados, abatidos aos quatorze meses. Pesquisa Agropecuária Brasileira, Brasília, v.32, n.10, p.1091-1095, out. 1997.

RIO GRANDE DO SUL. Normas técnicas para classificação de carcaças de bovinos e bubalinos para fins de enquadramento no "Programa Carne de Qualidade", instituído pela lei ${ }^{\circ} 10.533$, de 03 de agosto de 1995, do Governo do Estado do Rio Grande do Sul Diário Oficial do Estado do Rio Grande do Sul, Porto Alegre, v.54, n.188, p.42-43, 29 set. 1995. 